\title{
Mapping the Beach beneath the Street: Digital Cartography for the Playable City
}

\author{
Paul Coulton, Jonny Huck*, Adrian Gradinar and Lara Salinas. \\ Imagination, Lancaster Institute for the Contemporary Arts, The LICA Building, Lancaster \\ University Bailrigg, Lancaster LA1 4YW, United Kingdom. \\ * School of Environment, Education and Development, Arthur Lewis Building, The \\ University of Manchester, Manchester, UK M13 9PL.
}

\begin{abstract}
Maps are an important component within many of the playful and gameful experiences designed to turn cities into a playable infrastructures. They take advantage of the fact that the technology used for obtaining accurate spatial information, such as GPS receivers and magnetometers (digital compasses), are now so widespread that they are considered as 'standard' sensors on mobile phones, which are themselves ubiquitous. Interactive digital maps, therefore, are are widely used by the general public for a variety of purposes. However, despite the rich design history of cartography digital maps typically exhibit a dominant aesthetic that has been designed to serve the usability and utility requirements of turn-by-turn urban navigation, which is itself driven by the proliferation of in-car and personal navigation services. The navigation aesthetic is now widespread across almost all spatial applications, even where a bespoke cartographic product would be better suited. In this chapter we seek to challenge this by exploring novel neo-cartographic approaches to making maps for use within playful and gameful experiences designed for the cities. We will examine the potential of design approaches that can producte not only more aesthetically pleasing maps, but also offer the potential for influencing user behaviour, which can be used to promote emotional engagement and exploration in playable city experiences.
\end{abstract}

Keywords - digital cartography, neocartography, digital maps, game design, location based games. 


\section{Introduction}

The title of this chapter includes the phrase "The Beach Beneath the Street", which refers to the graffiti "Sous les pavés, la plage!" that appeared in Paris during the civil unrest of May 1968. This phrase is often translated as "Beneath the pavement-the beach!" (Wark, 2015) and is most often associated with the influential French avant-garde group the Situationist International (SI) who took part in the street demonstrations. The SI argued, in a similar vein to Henri Lefebvre (1991), for a right to view the city as a space for democratic possibilities, a social geography of freedom within which the rules of everyday life would be turned upside down and restored into a "realm for play" (Shepard and Gregory, 2011). One of the key concepts of the SI is 'Detournement' which is French for 'rerouting' or 'hijacking' and was used to describe the reuse of preexisting elements to create a new ensemble (Debord \& Wolman 1981). One of the main SI practices is the dérive (drifting), a technique of rapid passage through varied ambiances. Dérives involve playful-constructive behavior and awareness of psychogeographical effects, and is different from the classic notions of journey or stroll.

Psychogeography was first introduced into the SI discourse by Guy Debord in his essay Introduction to a Critique of Urban Cartography:

"Psychogeography could set for itself the study of the precise laws and specific effects of the geographical environment, whether consciously organized or not, on the emotions and behavior of individuals. The charmingly vague adjective psychogeographical can be applied to the findings arrived at by this type of investigation, to their influence on human feelings, and more generally to any situation or conduct that seems to reflect the same spirit of discovery."

The SI celebration of revolutionary play is revived in creative explorations of location-based technologies. These location-based practices often adopt the 'tracing while wandering' tactic of the dérive to map the city’s geography (Tuters \& Varnelis 2006). From media art to human computer interaction design, these situated practices 'bridge the digital with the analog' to 're-imagine urban space from the point of view human subjectivity' (Tuters, 2011. The concept of a 'Playable City' echoes the desire of re-appropriating and re-mapping our urban infrastructures as spaces of play and provides 
a useful lens through which we can consider the games and playful experiences discussed in this chapter.

Whilst there is a long history of street games (Culin, 1891) that could be explored within this chapter, in this research we concentrate on the games that have emerged through the utilisation of mobile phones and their infrastructures. In particular, we will focus upon the so-called 'Location Based Games' (LBG), in which the gameplay evolves and progresses in relation to the player's physical location. Inherent within this definition is a requirement for a player to be able to indicate their location during gameplay. However, whilst providing location is increasingly abstracted from the developer at a technical level it remains a complex process (Rashid et al 2006) and a wide variety of techniques have emerged. These techniques take advantage of either the mobile infrastructure, for example the Cell-ID, or sensing technologies embedded into mobile phones such as GPS receivers. Perhaps unsurprisingly, the earliest location based games such as Botfighters, Undercover, The Journey, and I Like Frank all used Cell-ID (Rashid et al., 2006) although its prevalence was relatively short-lived due to the emerging availability of GPS, initially through separate devices connected via Bluetooth and later through GPS chipsets being built into mobile phones. The most well known of the early GPS games is arguably Mogi from Newt Games, which achieved notable success in Japan (Licoppe and Inada, 2008). Other early games experimented with implied location solutions where players interacted with objects or systems that have a known location using such things as NFC (Coulton et al. 2006), Bluetooth beacons (Rashid et al., 2005), and visual markers (Chehimi et al., 2008).

Whilst all of these early games used location, they preceded the availability of mobile mapping services, and so used either bespoke maps of a specific area or adopted a radar style visualisation by simply showing reletive proximity to other game players or objects. Further, the resolution of these visualisations was severely restricted by the resolution of the mobile screens as illustrated in Figure 1 which shows the map for the location based game PAC-LAN (Ra- 
shid et al., 2006). This map represents a physical game space of approximately $0.5 \mathrm{~km}^{2}$ on a $128 \times 128$ pixel screen.
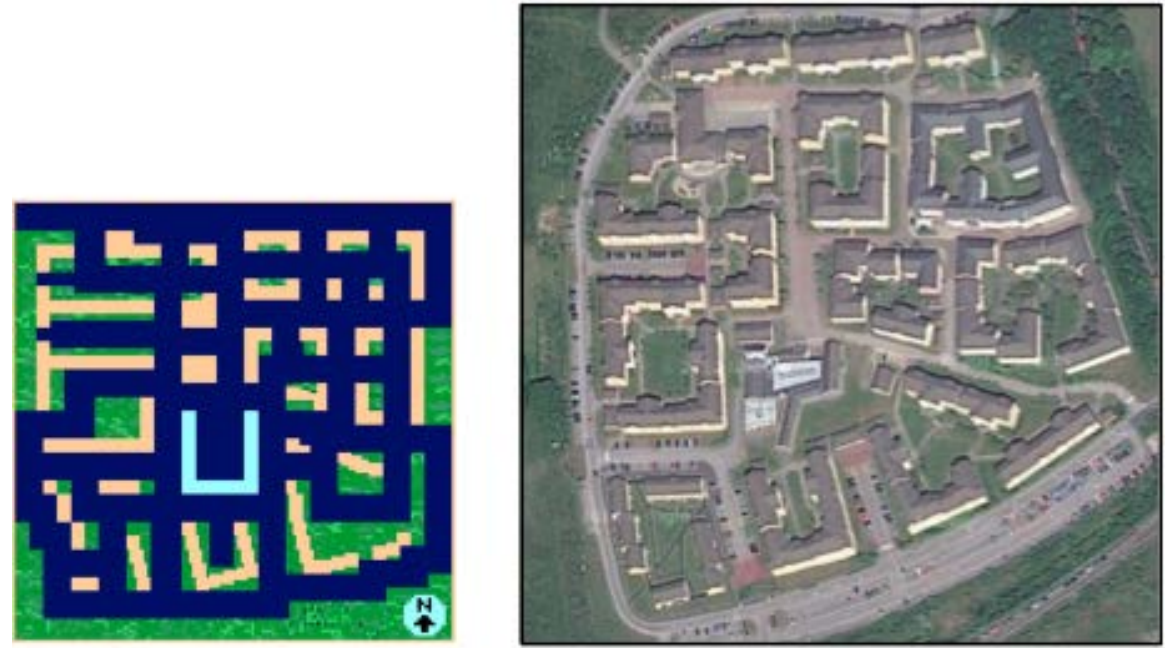

Figure 1 First PAC-LAN game map (left) and the area it represents (right)

Maps constitute a ubiquitous medium through which we understand, construct and navigate our surroundings (Ballatore and Bertolotto, 2015). As interactive digital maps have increasingly replaced paper maps, this "ubiquitous cartography" is quietly becoming part of our daily lives (Ballatore and Bertolotto, 2015). One of the major drivers for this was the launch of the Google Maps API in June 2005, initiating a wave of location based services and mash-ups. The release of Google Maps for Mobile in 2007 and its API in 2008 initiated a further developer frenzy and whilst other mapping services have appeared and subsequently disappeared, it undoubtedly remains the dominant mapping service. It is widely acknowledged amongst cartographers and map-makers that the advent of 'mobile maps' has had a profound impact upon the way in which we interact with mapping in general. "Map Readers" have become "Map Users" (Muehlenhaus, 2014), and their expectations have changed accordingly. Users no longer look at maps to find where they are, but rather they 'tell' the map where they are and expect the map to orientate itself around them (Clark, 2008). The generic nature of these 
maps, however, means that finding the geographical information relevant to the task at hand can often be a challenge for users, as they are faced with a 'default' map containing standard feature content that does not account for their specific needs or preferences (Wilson et al. 2010). In an attempt to combat this, map providers typically overlay their maps with information (often described as Points of Interest (POI) that may be of some relevance to the user, based upon stored information about them. Unfortunately, some this information will naturally be superfluous, and can lead to "spatial information overload”, whereby irrelevant feature content can actually hinder users, and in particular, task goals (Wilson et al. 2010).

Whilst tools such as Google Maps make it 'simple' to create digital maps, they provide very little scope for aesthetic customization beyond simply re-colouring map features. The market dominance of these maps have led to expectations amongst map users that a digital map will look and behave like a Google Map. A further expectation is that can be quickly produced and without the requirement for 'expert' decision making with regards to symbology, projection, scale and so on (Muehlenhaus, 2014). It is not surprising, therefore, that many cartographers have lamented the rise of these commercial mapping tools, which come with predefined projection, scale, typography, colour and symbology (Field, 2013). It is argued that such predefined digital maps are creating a global "blandscape" and a sense of "unauthordness" through the lack of detail and apparent "homogenization" of the landscape (Kent 2008). This unauthordness, also evident in location based games, is disappointing given that other types of game have a strong history of making extensive use of maps. The purpose of this chapter is to consider why this blandscape has been created, and how we might encourage designers that cartography can take a greater role in the creation of playable cities.

\section{Cartography - Neocartography}

It has been widely reported that the discipline of cartography has "struggled to keep up" with rapid developments in digital mapping 
technologies over the past quarter-century (Muehlenhaus, 2014), with each major technological development in map making (desktop GIS, web mapping, mobile mapping) being lauded as the "death of cartography" by many commentators (e.g. Wood, 2003; BBC, 2008). Indeed, it is claimed that many modern maps, particularly those on the web, are now produced "without cartography", as the projection, scale, typography, colour and symbology are all predefined by the map provider (Field, 2012). In an age where cartographic styles may be considered more as a commercial asset for brand recognition than a device for communication, there is little question that the craft of online map-making has developed without too much input from the discipline of cartography itself (Muehlenhaus, 2014).

It should be noted, however, that from the advent of thematic cartography in the early 1800's right up until the 1930's, mapping would be undertaken by architects, engineers and designers; not those specifically trained in map design (Muehlenhaus, 2014). Prior to this, maps were purely objects of reference, and only used for demarcating property ownership, state territory, urban layouts and war (Pickles, 2004). The word 'cartography' is, in fact, a relatively new term: it was coined as a Portuguese neologism in 1839, and only appeared in the Oxford Dictionary in 1859 (Wood, 2003). Cartography only developed as a field of academic interest following the Second World War, during which it was realised that maps were not only useful tools of objective visualisation, but also powerful rhetorical tools that could be used, for example, to spread propaganda (McMaster and McMaster, 2002; Muehlenhaus, 2014). It may be considered, therefore that maps have in fact been produced "without cartography" for the vast majority of their history, with only a brief period between the 1930's and the 1980's where institutionalised cartographic conventions prevailed. This view is supported by Wood (2003), who describes the conflation of map-making and cartography as "at best anachronistic, at worst unpardonably presumptuous". Modern developments in the field of digital neocartography, may also be separated from the 'traditional' body of academic cartography, with a focus that is much more seated in the practices of design than cartography (Wood, 2003). 
Muehlenhaus (2014) questions whether Google Maps has "revolutionised" or "euthanized" map making, with the ubiquity of Google Maps now effectively meaning that people are coming to expect that all maps should look and behave like Google Maps. The result of this is, of course, that many more maps are created using services from commercial mapping providers such as Google. In turn, this has further reduced the demand for designed-for-purpose cartographic products, leading to the subsequent reduction in the development of cartographic tools and software, arising from insufficient demand. In their place we have seem a practice emerge in which the regular creation of maps is performed by almost anyone, with little regard for data, design philosophies, or principles of mapping (Field, 2012). Furthermore, the ability to make such maps more easily has, in many cases, led to the expectation that should be made quickly, once again at the expense of the rigorous design process required for a high-quality cartographic product (Field, 2013). This results in an over-reliance upon "dumb defaults", and the impression that the "art" of map-making is being lost as all maps start to look the same (Field, 2013).

Nevertheless, mapping, like writing, can be done lucidly and elegantly (Monmonier, 1993), and there is little doubt that recent developments in the field of digital cartography have permitted the development of approaches to mapping that are able to communicate information more effectively, and even have the potential to affect the behaviour of the map reader (Huck et al. 2015; Huck et al. 2016; Gradinar et al. 2016). Irrespective of the specific purpose of a map, the era within which it was created, or the media or technology with which it is produced, the primary goal remains the same: a map seeks to communicate some form of information (Field, 2012; Muehlenhaus, 2014); and for this to be achieved, modern digital cartography must strike a balance between art, science and technology in order to be effective (Field and Demaj, 2012). A beautiful map based upon miscalculated, poorly measured or otherwise incorrect data may be considered to be of little utility; and conversely an ugly or unclear map, however accurate, is unlikely to inspire the confidence of the map-reader, also limiting its utility (Wright, 1977). 
In recent years the reported shift towards the paradigm of neogeography (Turner, 2006) has been accompanied by much discussion about the emergence of neocartography (Kraak, 2011). Whilst the definitions for terms such as these can be somewhat nebulous, Kraak (2011) describes a "working definition” for neocartography as: “a limited set of loosely coupled simple guidelines that allow an adequate visualization of large amounts of diverse data sets". For the purposes of this chapter, neocartography may be considered as the area of cartography that deals with the automated rendering of multiscale data using style-sheets or similar rule-based approaches, with little or no manual intervention from the cartographer or designer. This contrasts with the traditional approaches to cartography, in which the cartographer or designer would interact manually with many map elements, in order to produce a map that would normally represent a defined region at a given scale. Neocartography, therefore, may certainly be considered to encompass those technologies that produce tiles of data at multiple scales to populate the 'slippy map’ interfaces of neogeographical web maps such as Google Maps, Bing Maps and OpenStreetMap. Data sources such as OpenStreetMap, technologies such as Mapnik, and companies such as Stamen Design and MapBox have been instrumental in bringing neocartography into popular use, through the release of open data and open source software that allows individuals to create and implement their own neocartographic style-sheets. These developments have, in turn, allowed the creation of exciting new possibilities in the production of novel digital mapping content (Huck et al. 2015).

\section{Mapping in Games}

Arguably, the history of games is as old as the history of maps, with evidence of board games being played for more than 5000 years (Whitehill 1999). Maps have played an important role in many games throughout history; treasure maps in board games, schematic reality-inspired maps in Monopoly, maps of fantasy worlds in video games, and so on. Perhaps the most well known, however, is Chess, which, like Draughts and Go, was once a real world simulation in 
which players assume the role of an army leader charged with the objective to overcome an enemy and conquer territory (Ahlqvist, 2011). The primary role of maps in games is to support the game mechanics and rules. Many of the early games employed very simple and abstract maps, replacing 'realistic' looking cartography with very basic representations of territory that were much easier to replicate (e.g. the chequered pattern on a chessboard). This changed in the mid-19th century when advances in lithography and production techniques allowed games to be printed in large commercial quantities and coincided with an expansion of games away from war-like simulations to encompass a wide variety of narratives (Ahlqvist, 2011). Whilst the history of maps in physical games deserves a more detailed discussion, in this chapter we are concerned with computer games and so such a discussion is, unfortunately, beyond its scope.

In the case of early computer games, it is often tempting to begin the discussion with arcade and console games such as Pong, Space Invaders, Lunar Lander, and so on. The advent of maps in computer games predate these with the 1975 text based game Adventure and the first Multi User Dungeons (MUDs) invented by Roy Trubshaw and Richard Bartle in 1978, which are arguably the first significant developments of mapping in computer games. The main component of these first MUD's was a database that was used to define the rooms, objects, commands etc., which was defined in a separate file from the main game code. This meant that players were able to add new features, or otherwise alter the game, by editing these files. Despite the games providing either no visual information or graphics based upon ASCII characters (as shown in Figure 2), the games themselves, along with the players' modifications, perhaps best represent the first examples of cartography emerging within computer games. This practice of altering games became known as 'modding', and persisted as maps continued to became integral within computer games. This was particularly clear with the emergence of First Person Shooters (FPS) such as Doom in 1993, which allowed third parties to create custom levels; effectively designing their own maps. Modding became such a popular activity that level editors appeared in early 1994 and indeed the practice became the entry point for a number of future game designers (Sotamaa, 2010). 


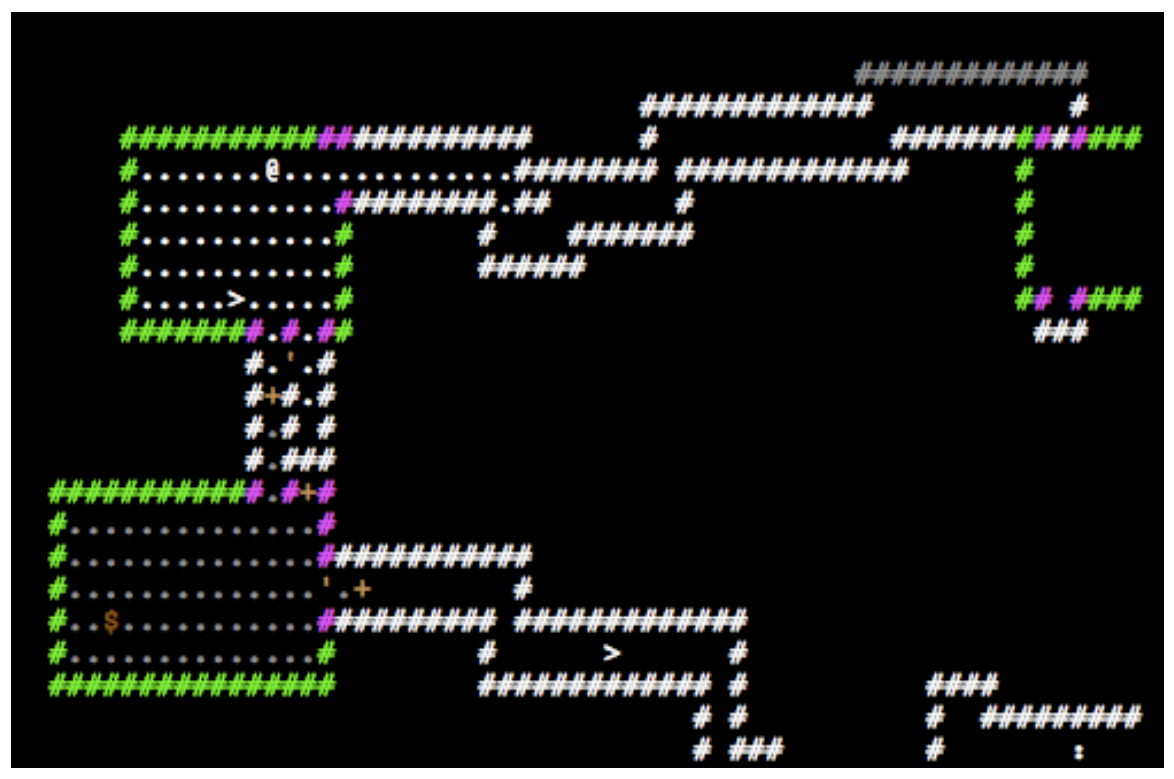

Figure 2. Example of an ASCII MUD Map

Mapping has seen a resurgence in the most recent generations of video games, which have been described as "the bold future of cartography" (Garfield, 2012), and have often been cited as a notable exception to the perceived stagnation of map production. Games such as 'Grand Theft Auto V' by Rockstar Games (2014) and 'Elder Scrolls V: Skyrim' by Bethesda Game Studios (2011) comprise not only detailed 3-dimensional 'open world' maps to explore within the game, but also 2D on-screen maps for navigation, and even accompanying paper maps. 'Skyrim', for example, comes with a fold out stylised map on textured faux-parchment and a game guide that includes 220 pages of maps, representing the work of many digital cartographers (Garfield, 2012). Further, cartography still dominates much of the current modding activities with numerous sites offering maps for popular games such as Half Life, Counterstrike, and Minecraft. Given this interest, maps can be seen as a vital component of modern game production. Having discussed maps in games more generally, we will now consider the role of maps in games designed to facilitate a playable city. 


\section{Mapping in Location Based Games}

The concept of 'location based' game came to the fore with the removal of 'Selective Availability' from the GPS network in May 2000. The resulting proliferation of hand-held GPS receivers meant that, for the first time, the general public had access to their own location via the GPS network. Geocaching, arguably the first digital location based game, was first played that same month, with players initially using GPS-derived coordinates to hide and seek 'caches' (reminiscent of the traditional orienteering game letterboxing). The coordinates of caches were provided in online lists, and the 'caches' often took the form of a waterproof container containing a logbook as well as some trinkets which players are encouraged to trade through the caches. As GPS receivers started to be embedded into mobile phones, a dedicated application for Geocaching was created that included a Google Map with icons to assist players in finding 'caches'. Nevertheless, similar games such as Munzee have been much faster on the uptake of emerging smartphone technologies, including QR codes and NFC tags, and as a result are attracting many former Geocaching players. Today there are more than 2.5 million geocaches in over 180 countries and more than 10 million registered users on Geocaching.com.

As already identified, the map component of the Geocaching mobile app is a Google Map. Whilst, prior to the availability of commercial web mapping services, some games created their own customised maps (e.g. Rashid et al. 2006), Google Maps now dominate in location-based gaming. For example, the mafia style type of game TurfWars $^{1}$; the role-playing games such as Parallel Kingdom ${ }^{2}$ and Global Supremacy3; and the academic research game Urbanopoly (Celino, 2012) all rely upon Google Maps. The reason for this is clear; Google Maps provide a familiarity in appearance and function that has come to be expected by users, and an ease of implementation that is attractive to developers. Nevertheless, there are some ex-

\footnotetext{
$1 / /$ turfwarsapp.com

$2 / /$ www.parallelkingdom.com

3 //www.globalsupremacyapp.com
} 
amples where bespoke maps have been implemented within location based games. The now discontinued Shadow Cities ${ }^{4}$ is one such example, as is the augmented reality game Ingress ${ }^{5}$, which makes use of the Google Maps data to create a personalised map for each player. However, such games are few and far between and, whilst their bespoke maps do contribute positively to the game aesthetic, they make no attempt to explore the impact that novel approaches to map design could have upon the gameplay.

Whilst not Location Based Games per se, activity fitness tracking applications are increasingly making use of digital maps. Once again, the dominance of commercial mapping providers such as Google Maps is demonstrated by applications such as Strava ${ }^{6}$, Endomondo $^{7}$, Runtastic ${ }^{8}$ and Nike Plus ${ }^{9}$. To enhance the user's immersion levels, these applications have been augmented with additional content. Examples of this include Runtastic, which includes a "Story Running" activity ${ }^{10}$, where users can listen to a diverse range of stories intended to enhance the running experience; and Zombies Run ${ }^{11}$, which adds a narrative into 'missions', enhancing the activity with game like adversaries such as zombies 'chasing' the player, or the achievement of in-game quests that are required to 'build your base' and 'defend your community'. Finally other applications, such as Swarm (formerly Foursquare), may be called 'quasi-games' because they do not have very well established rules so may be considered as occupying a 'middle-ground' somewhere between social networking tools and games (Lammes, 2011). Whilst such quasi-games exhibit play-like elements, their status as games is not universally accepted (Deterding et al. 2011) and the majority of these utilise the same navigational aesthetic.

\footnotetext{
4 //www.engadget.com/tag/Shadow-Cities

$5 / /$ www.ingress.com

$6 / /$ www.strava.com

$7 / /$ www.endomondo.com

$8 / /$ www.runtastic.com/en/apps/runtastic

9 //www.nike.com/gb/en_gb/c/running/nikeplus/gps-app

$10 / /$ www.runtastic.com/en/storyrunning

$11 / /$ zombiesrungame.com
} 
Having highlighted the importance of maps in games and a requirement for greater consideration to the design of maps in location based games and playful experiences we now consider how this can be practically achieved.

\section{Designing Maps for Playable Cities}

We believe that digital map aesthetics can be optimised for a variety of given purposes and in this section we present two case studies that explore this through the novel cartographic techniques of ' $f e a-$ ture abstraction' and 'dynamic visual hierarchies' in order to demonstrate how aesthetic decisions can alter the experience and even behaviour of users (Gradinar et al 2016).

\section{PAC-LAN Zombie Apocalypse: Feature Abstraction}

The original PAC-LAN location based game was created in 2006 to consider the possibilities arising from the emerging combination of mobile phones with Near Field Communications (NFC), at the time using the general name of Radio Frequency Identification (RFID), to create new location based experiences (Rashid et al., 2006a). The game was a novel implementation of the arcade classic Pac-Man, which was chosen as it allowed a comparison with other location based versions of the game developed around the same time, such as 'Pac Manhattan' and 'Human Pac-Man' (Rashid et al., 2006a). Principally, the game investigated using NFC as an alternative to GPS as the method of determining location by requiring players to tag physical objects at known locations.

As the game proved successful and technology has matured considerably in the intervening period, a new version of the game was developed in 2015 with the focus much more on the overall experience rather than the interaction with the technology (Gradinar et al., 2015). PAC-LAN: Zombie Apocalypse was built using the Android platform to allow the game to be distributed 'in the wild', as Android 
supports the greatest number of handsets with on-board NFC readers. Similar to the original PAC-LAN game, the 'pills' that Pac-Man collects during the original arcade game were recreated as physical items using yellow and red Frisbees. The pills also included straps so they could be attached to the physical environment (e.g. lamp post, drainpipes, trees, etc.) and an NFC tag, allowing access to the name and location of that pill via the game system.

The game supports five players, each of which adopts a particular character persona (indicated by wearing a character hat) and are identified by a NFC tag along with an Android mobile device running the game application. One player takes the role of PAC-LAN, whose purpose is to tag all of the pills in order to earn points; and the remaining four players take the role of the Zombies, whose purpose is to 'infect' the PAC-LAN player by scanning their NFC character tag. As with the arcade version, there are a number of special 'power pills' located within the game arena which, when tagged by PAC-LAN, enable the player to 'neutralise' the Zombies and forcing them to return to the starting location of the game to 're-spawn'. A key aspect of the game design is that all of the main interactions within the game (such as tagging pills or characters, for example) occur physically and do not require any direct interaction with the interface of the mobile screen.

Before starting a particular game of PAC-LAN the game space needs to be set up, which involves positioning the pills both physically in the landscape and digitally on the map, which is undertaken within the mobile application. There are 3 types of pills in the game:

1. The base pill: This is the location where all players start the game and where players 're-spawn' after being neutralised (Zombies only) or if their 'roaming time' expires (all players).

2. Check-in pills: These pills update the last tagged location of all active players in the game database, as well as replenishing their 'roaming time' when they tag it, allowing them to stay in the game for longer.

3. Power pills: These pills give PAC-LAN the ability to 'neutralise' Zombies whilst preventing the Zombies from being able to 'in- 
fect' PAC-LAN. They also replenish the 'roaming time' and extra time for 'infecting' zombies. These pills are only distinguished on the PAC-LAN's UI as for Zombies they function the same as check-in pills.

Once the physical game space has been set up, each player tags the base pill to start their game. The PAC-LAN player begins play immediately (Figure 3 left), whereas each Zombie player has a predefined waiting time of between 100 and 40 seconds that must expire before they can join the game (Figure 3). This gives the PACLAN player time to safely exit the immediate vicinity of the Zombies before they can give chase.
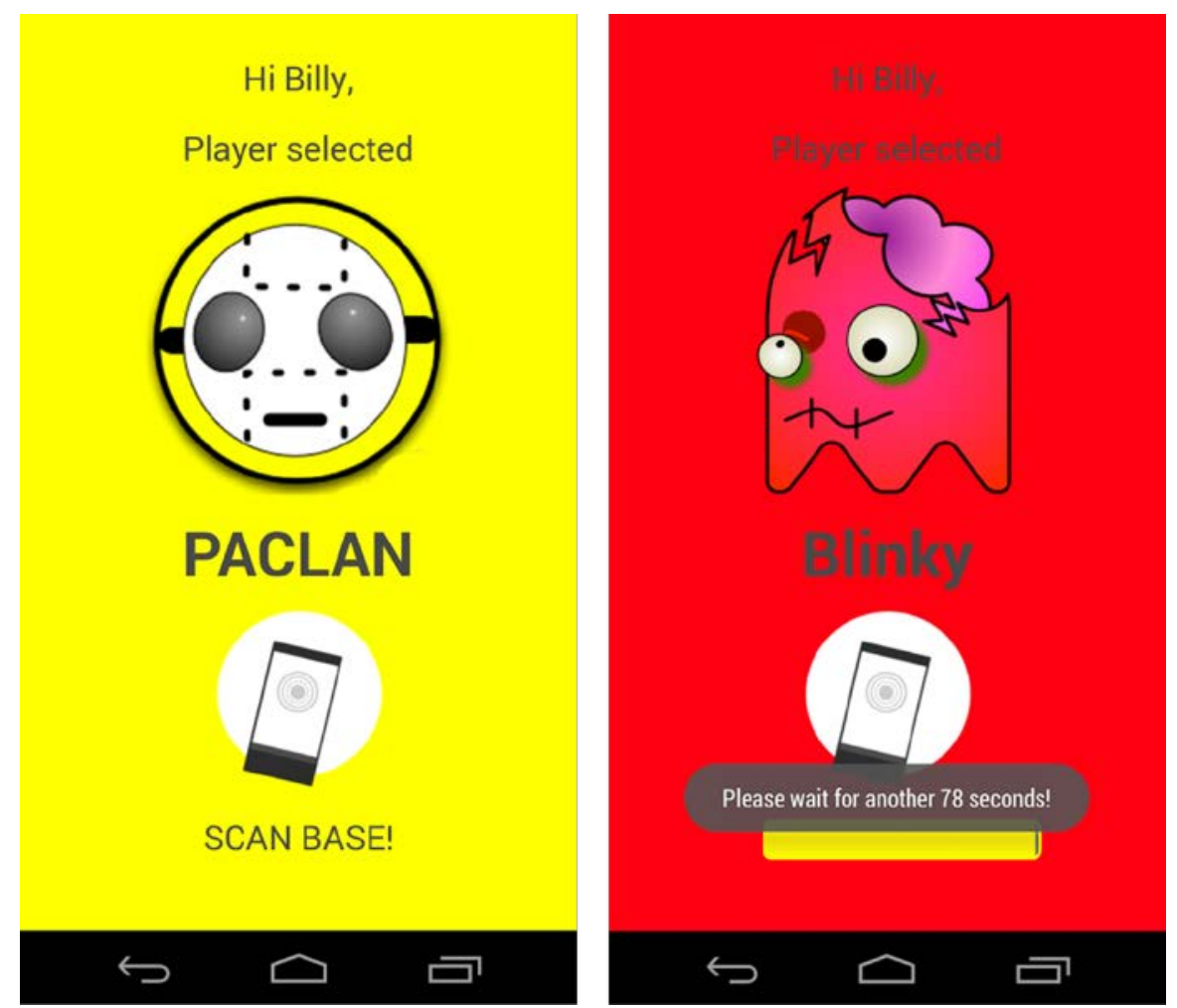

Figure 3. PAC-LAN entering the game (left) and Zombie entering the game with a countdown for 'waiting time' (right). 
The game user interface (UI) shown in Figure 4 consists of a map (considered in detail in forthcoming section) depicting the location of all pills, paths and buildings, and a decreasing progress bar ('roaming time') that represents the maximum time that a player can take between tagging pills before being forced to return to the base. Whilst returning to the base, the player is inactive and so no interactions with the UI or game pills is allowed until 'the base pill' is scanned again. When a player tags a pill, the 'roaming bar' is replenished and the UI refreshed by showing the location of the last pill tagged by each active player, as well as changing the colour of the pill to grey to indicate it is no longer worth any points. Each player's interactions with the pills are visible only on their individual UIs. The player can return and 're-tag' a grey pill to replenish their 'roaming time' and to update the last tagged location of all other active players, though they will not receive any more points for doing so.

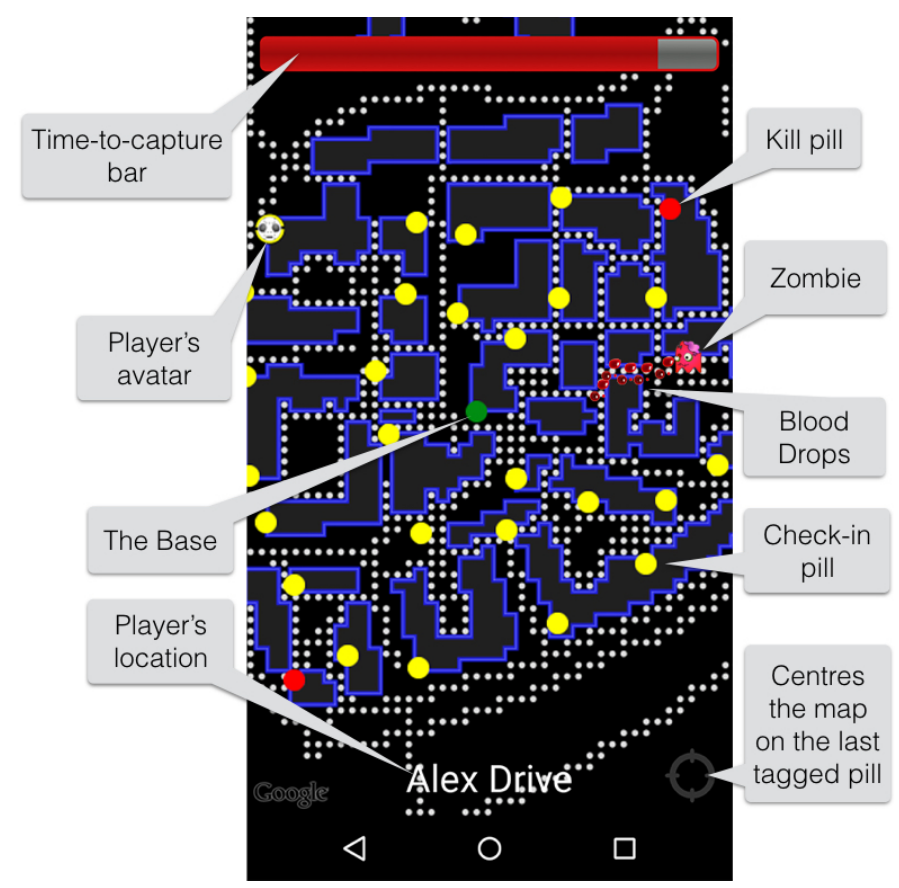

Figure 4. PAC-LAN Game User Interface 
Whilst the previously described features change the sequel (2015) considerably from the original PAC-LAN game (2006) it is the digital game map that is the primary differentiator. In particular the introduction of the smartphone instead of the original feature phone permitted the design of a high-resolution, dynamic 'slippy' map that could be generated in real-time based upon real geographical data, as opposed to the low-resolution static map that had been used in the previous version.

In the design of the map for this game, three design goals were considered:

1. Promote immersion into the game through the use of a suitable aesthetic.

2. Perform well in the context of a mixed reality LBG (running, outdoors).

3. Encourage players to navigate 'head-up' rather than 'head-down'.

In particular, the third design goal was of key importance to this game, as the research-goal was to explore the Dichotomy of Immersion (Huck et al, 2015): that peculiar situation created in LBG's whereby a player's attention is constantly divided between the physical world within which the game is being played (the physical world), and the screen of their mobile device (the digital world). The split of attention between the physical and digital game components is usually dominated by interaction with the screen at the expense of interaction with the landscape, which can limit emotional engagement with (and therefore immersion into) the LBG (Zhang et al, 2012).

This design goal was approached by the use of 'feature abstraction' as a cartographic device. It was hypothesised that a small amount of abstraction in map features may encourage players to 'look up' more and verify what they see on the map against their physical surroundings, thus increasing their engagement with their physical surroundings. This is in contrast with the use of a more traditional (precise) map, which will not require validation against the landscape; or a map that is 'too abstract', which may be too difficult 
to read quickly whilst playing, thus increasing interaction with the screen at the expense of the landscape.

Four of the cartographic solutions to the above design goals will be presented. The maps have been all created using data derived from OpenStreetMap using PostGIS, and have been rendered using Mapnik. For the purpose of comparison, all maps are shown at the same standard orientation (north at the top), zoom level (17, equivalent to a geographic scale of 1:4514) and extent (showing part of the Lancaster University campus). None of the figures include a legend, north arrow, scale bar or similar, as such features are not typically provided in LBG's. In-keeping with the above design goals, all of the maps are highly limited in terms of the number of features they contain, with only 4 or 5 feature classes typically included to facilitate easy reading. For the same reason, augmentations such as labels and points of interest have also been omitted from all of the map designs.

The first map presented is the Pac-Map (Figure 5), which is designed primarily to match the aesthetic of the classic Pac-Man arcade game (Namco, 1980). Abstract feature representation has been achieved by generalising all nodes to the nearest $10 \mathrm{~m}$, and forcing vertices to be oriented either north-south or east-west: resulting in abstract features that also contribute to the game aesthetic. There are only 4 feature classes included in the map: 'building', 'path', 'trees' and 'hazard', all of which are rendered using styles directly inspired by the Pac-Man game. Trees and buildings are drawn in the same blue as the Pac-Man maze, and a complementary red has been used to mark out hazards. In order to ensure that the dark palette employed by this map performs well outdoors, the lines in the map are thick, with very fine white lines drawn into the blue and red in order to increase their contrast with the black background. Pathways (including roads and footpaths) have been marked out with white dots, once again to gain contrast with the dark background, whilst also reflecting the 'pills' that Pac-Man collects from within the maze in the original game. 


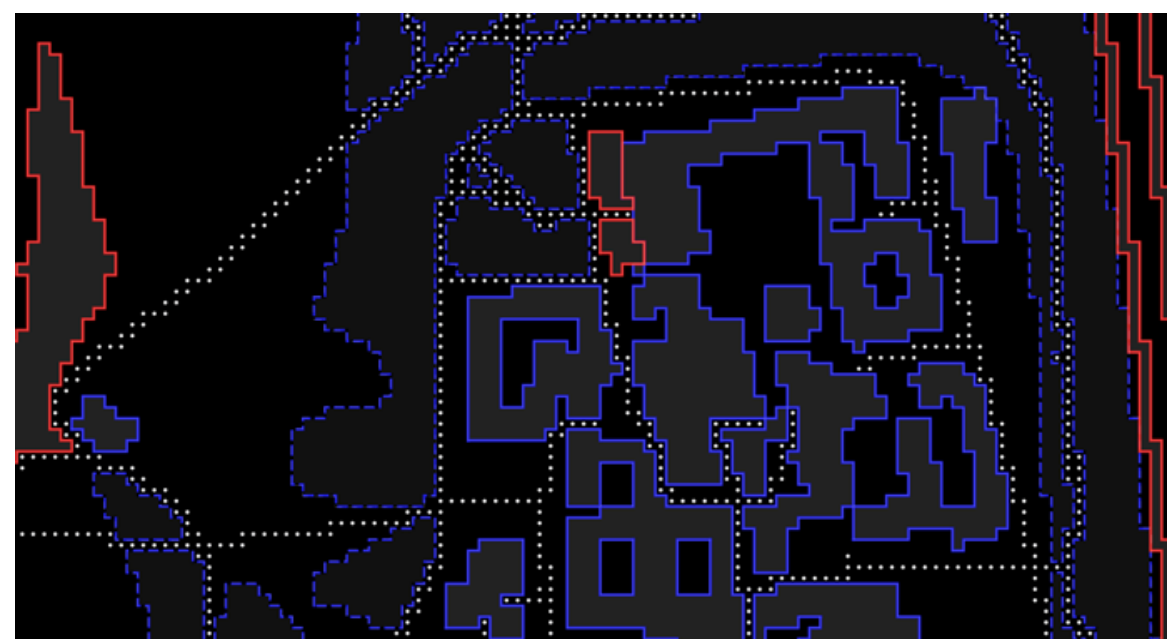

Figure 5. The 'PAC-MAP' design for PAC-LAN.

The second, RPG Map (Figure 6) is inspired by the 'classic' Role Playing Games (RPG's) of the 1980's and 1990's. The data has been abstracted into a grid of $20 \mathrm{~m}$ cells, each of which can only contain one of five feature classes: 'building', 'road', 'water', 'trees' or 'hazard'. Cells were then dissolved into contiguous areas of each data type, and coloured using tiled textures collected from freely available online sources. The use of a coarse $20 \mathrm{~m}$ grid gives this map a greater level of abstract feature representation than the Pac-Map, therefore making it more difficult to rely upon for navigation, in order to investigate the effect that this has upon the player's interactions during gameplay. The coarse grid, RPG-style textures and playful features (e.g. the use of a 'lava' texture to denote hazards) lend a definite 'game aesthetic' to the map, but in less-specific manner to the Pac-Map, permitting exploration as to the effect of this upon players' perceived level of immersion. 


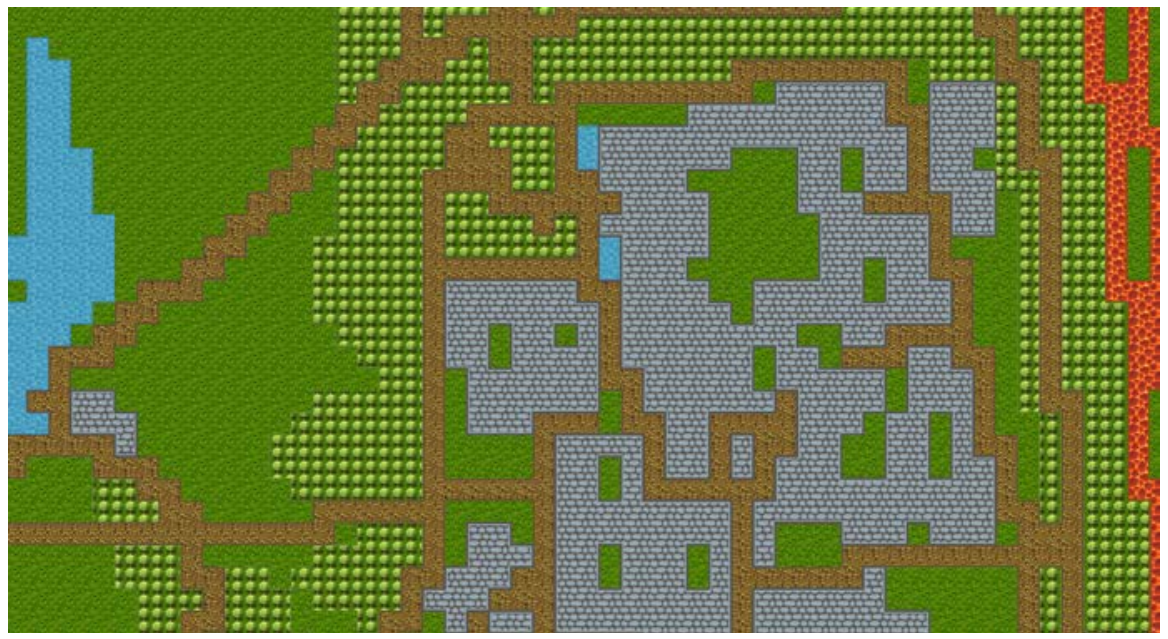

Figure 6. The 'RPG Map’ design for PAC-LAN.

Thirdly, the 'Sketchy Map' (Figure 7) employs 'sketchiness' as an approach to abstract feature representation, acting to obscure the precise position and shape of geographic features. The 'hand-drawn' or 'sketchy' effect on the polygons has been achieved by a combination of polygon smoothing, line smoothing, multiple-overlay and image composite operations in order to give the impression that they have been drawn using felt-tip pens (akin to the approach first suggested by Ashton, 2012). Conversely, the line features were simplified using the Visvalingam-Whyatt line generalisation algorithm (Visvalingam and Whyatt, 1993), and overlaid using transparency and image composite operations in order to give the appearance of having been drawn using highlighter pens. The main difference with this approach is that the level of abstraction varies from feature to feature as opposed to being uniform across the dataset as is the case in the grid-based approaches, which may prove more disorientating for users. The 'hand-drawn' aesthetic promotes a 'playful' feel to the map, but without specifically portraying a 'game'. 


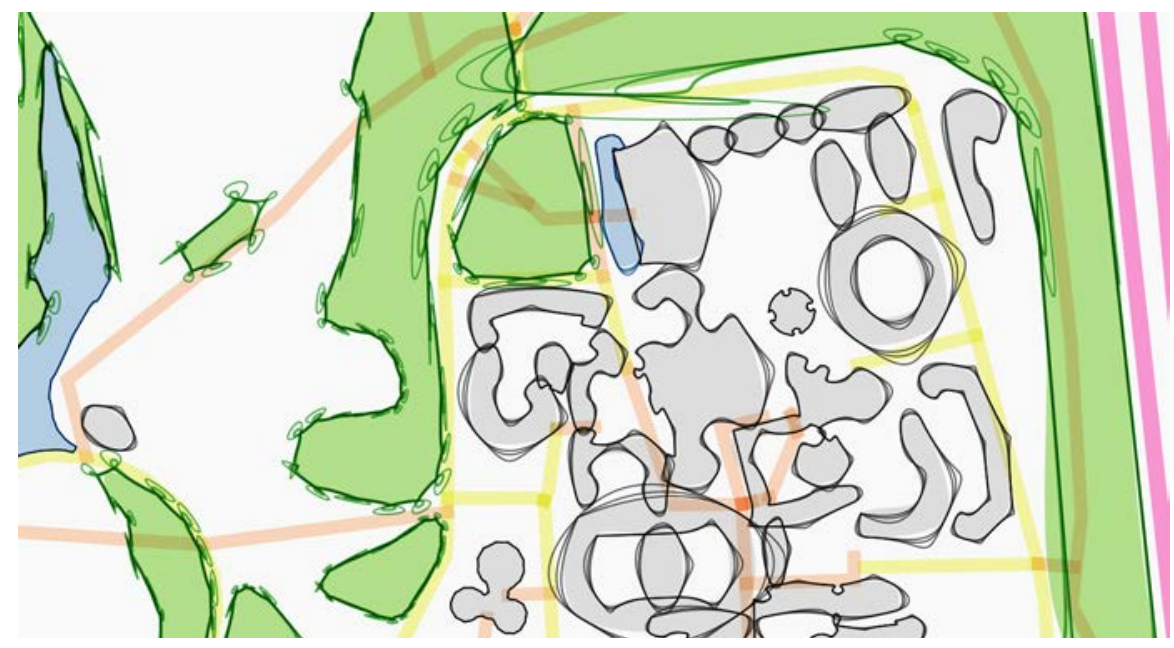

Figure 7. 'Sketchy Map’ design for PAC-LAN.

Finally, the 'Anti-Glare Map' was created which includes no spatial ambiguity whatsoever, and instead is characterised by a triadic colour scheme in order to maximise the contrast between all map features. This map is designed as a benchmark from which players could evaluate the other three maps.

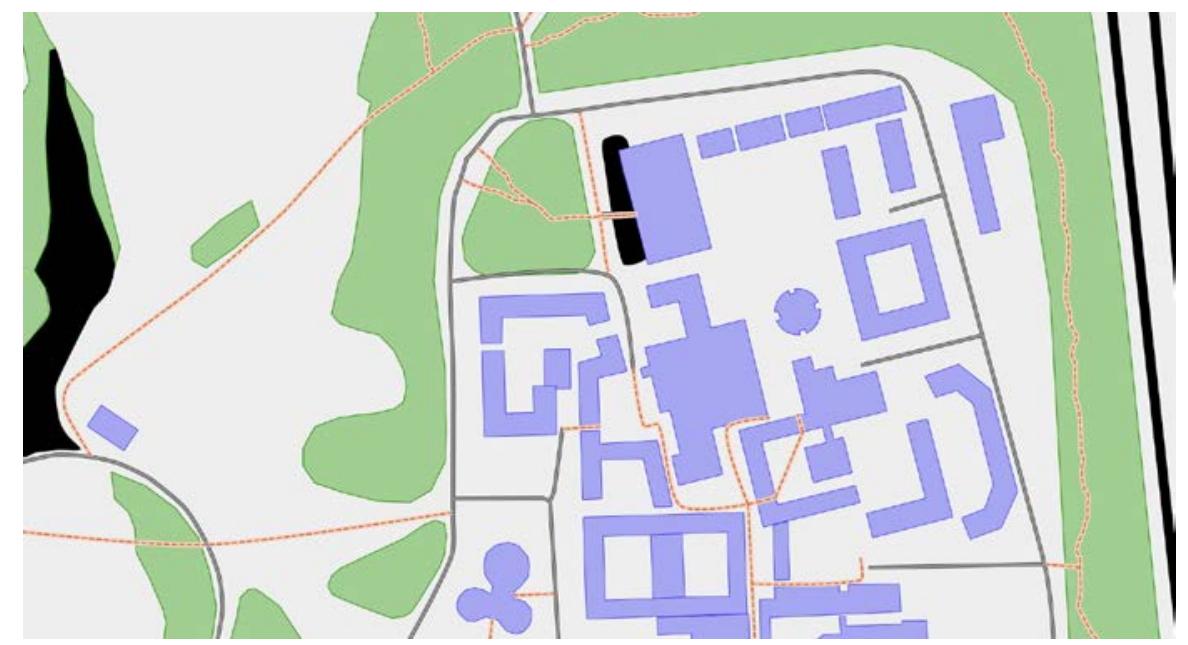

Figure 8. The ‘Anti-Glare Map’ design for PAC-LAN. 
These maps were tested with 8 players that were asked to play a short custom-built LBG that required them to navigate between 20 physical objects (the PAC- LAN game pills) with 5 objects per map type. The initial map was chosen at random and then changed for another randomly selected map after every fifth pill was correctly tagged, in order to allow the assessment of each map. The LBG tracked players' progress using GPS and required them to hold down the volume rocker of the mobile phone in order to view the map. This mechanic also means that players' interactions with the map were logged alongside GPS-derived location for later analysis using space-time prisms (Coulton et al. 2008) as shown in Figure 9. In this figure the base map is the physical area and time is represented vertically with the location of the pills shown in yellow and blue and red indicating whether the map is visible or invisible to the player respectively. Following play, all eight players were also given a semi-structured questionnaire and interview about how each map influenced their level of interaction with the screen and their surroundings.

The results of trialling the maps concluded that PAC-MAP style map (Figure 5) was universally preferred by the participants and was also the most suitable of the maps for the promotion of 'head-up' navigation in the game. The combination of the PAC-MAN gaming aesthetic and moderate feature abstraction promoted the game play whilst encouraging the player to validate what they saw on the map against the physical landscape. This result is interesting if viewed from a purely utility and efficiency perspective, which would clearly favour the Anti-Glare map and highlights the desirability for greater utilisation of cartography within location based games. 


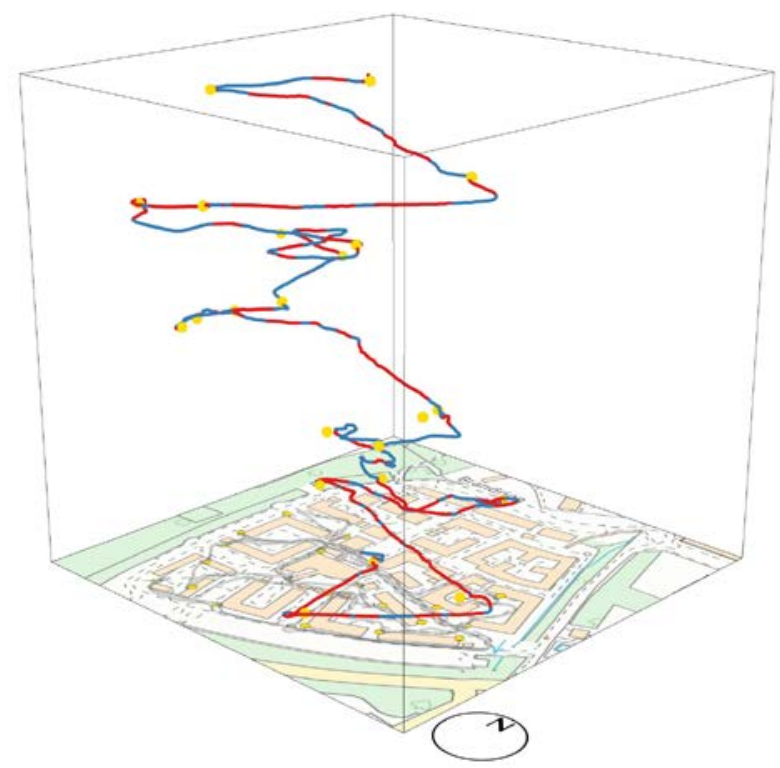

Figure 9. Space Time Prism of Map Trial Instance

There are also other deliberate design features that obscure parts or the whole of the UI at different times during the game, in order to encourage the player to be less dependant upon the screen. Firstly, The 'Fog of War' (FoW) shown in Figure 10 is a game feature controlled by the 'roaming time'. It consists of incrementally obscuring the map with a white 'fog', thus making it more difficult to read. There are four different levels of 'fog', with each level obscuring a greater area of the map as it incrementally 'creeps in' from the edge of the screen towards the centre. To further aid the player's awareness of the passing of the 'roaming time', each level of the FoW is associated with a haptic feedback, with handset vibrations informing the player of their remaining 'roaming time' without the requirement to check the screen. Secondly, 'Screen Blanking' is designed to discourage over reliance of the player on the mobile screen. It consists of turning the whole mobile UI into a black screen after 5 seconds from the last action the user performed. There are two types of actions that make the screen fully visible again: either the player tags a pill or player, or explicitly requests access to visualise the game in- 
terface by tapping the mobile screen. The latter action displays the UI in its current form, and so does not remove the FoW. It is important to note that screen blanking and the FoW are not mutually exclusive.
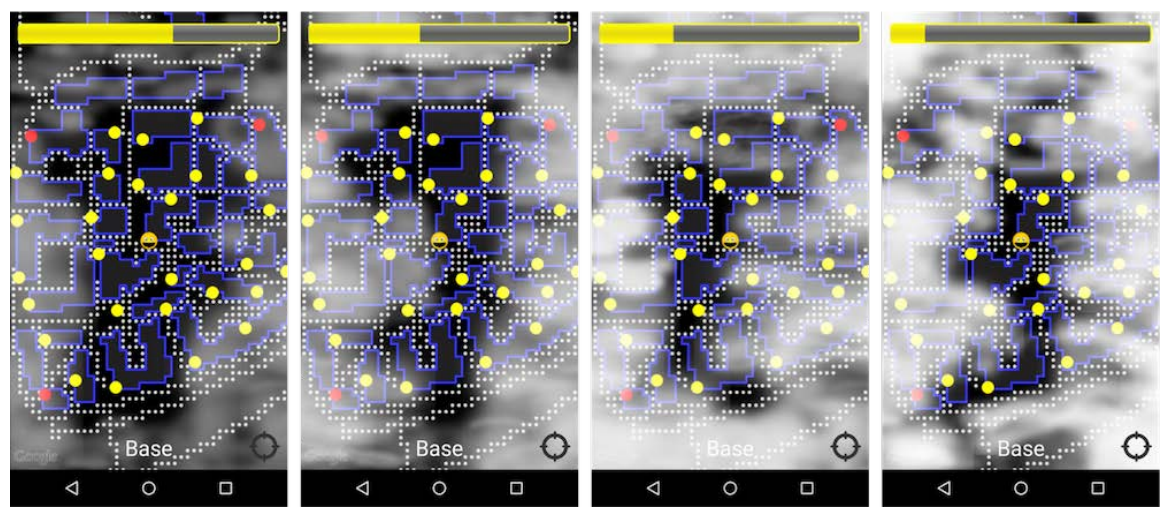

Figure 10. Fog of War operating on four levels on the UI

An additional 'Blood Drops' feature is used to assist the PACLAN player in finding Zombies whilst under the effect of a Power Pill, giving a slight advantage in their attempt to 'neutralise' a Zombie. As soon as the PAC-LAN player tags a 'power pill', a virtual trail of blood drops is displayed on PAC-LAN's map, which shows five GPS positions recorded after each Zombie last tagged a pill. Given that the PAC-LAN player does not know how long ago the Zombies last tagged a pill, this information indicates their initial direction of travel from their last tagged pill, but does not give away their location with any certainty. The game is finished when either PAC-LAN tags all the pills or is tagged by one of the Zombies and the winner is calculated based on the total points gained during the game. When the game finishes, the mobile application displays a leader board showing all five players and their scores.

Overall this study illustrates the benefits that designing maps with a particular aesthetics can have on playable city experiences and in the following we consider how hierarchy of the maps could also be used to change the nature of these experiences. 


\section{Paths of Desire: Dynamic Visual Hierarchies}

Map design revolves around the need to satisfy a particular communication goal (Field 2013) relative to a given task. For commercial mapping services such as Google Maps, this goal is primarily to act as a road atlas. It is arguably inappropriate, therefore, that these maps are often used for a wide range of purposes without due attention being paid to the suitability of the map for the task at hand. For example, many traditional paper tourist maps represent selected POI around a city but frequently sacrifice scale and completeness in favour of adopting a visual style that matches the image of the city they are trying to portray (e.g. an historic city), and focus upon the features of the city which would be of interest to tourists (e.g. museums).

Previous research has demonstrated that map design can have a significant effect on route selection, and that the visual hierarchy of roads and footpaths is one of the primary influencers (Field and Demaj 2012). In this example, we present a novel Android application called 'Paths of Desire', which unlike traditional digital maps has a cartography that can be dynamically adjusted in real-time, modifying the visual hierarchies of paths (including roads, footpaths etc.) and POI in order to try to encourage visitors explore the city. It is intended that the varying visual hierarchies will encourage users away from main routes and motivate exploration of areas that would otherwise be ignored.

Experimentation using this design aim has focused upon the city of Lancaster in the United Kingdom, which is a small historic city in the North West of England. Lancaster is dominated by a main pedestrianized street that runs through the town center and a circular trunk road that encloses the town center. Both of these features significantly influence the flow of traffic and pedestrians, as they move through the city. These are shown in Figure 11, along with the major points of interest and other often overlooked tourist attractions. 


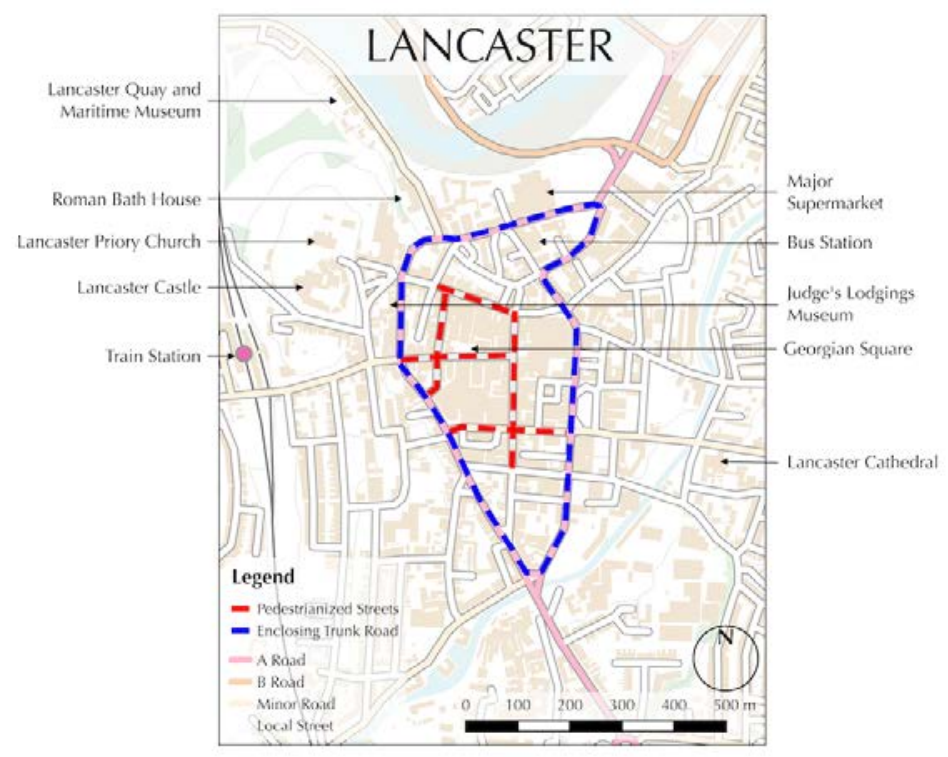

Figure 11. A map of the city centre of Lancaster in the UK, illustrating the limited areas of the city commonly populated by visitors $^{12}$.

Lancaster City Council has been actively engaged in attempts to attract both residents and visitors to other parts of the city through outdoor art exhibitions, music festivals, and the installation of new street furniture. Whilst these efforts have had some limited impact upon the problem, so far there have been no attempts to address the use of digital maps, which is the dominant way in which new visitors are likely to explore the city. The aim of this project is to consider alternative design solutions and determine the extent to which novel digital map design can be used to promote tourism in undervisited areas of the city.

To this end, an Android application has been developed to act as a 'digital tourist map' of the city. The design goal of the map is to promote exploration within the city by using 'dynamic visual hierarchies' to make features more or less attractive depending upon how many other tourists are currently located there (determined from the

${ }^{12}$ Contains OS data (C Crown copyright and database right (2015). 
GPS location of other app users). The map design used in the application is defined by an XML style-sheet that is downloaded from a server at launch. This allows for dynamic updates of the style-sheet without the need to push updates of the application to users devices. The initial map style guide was designed by the Manchester-based digital design agency Magnetic North, and is shown in Figure 12.
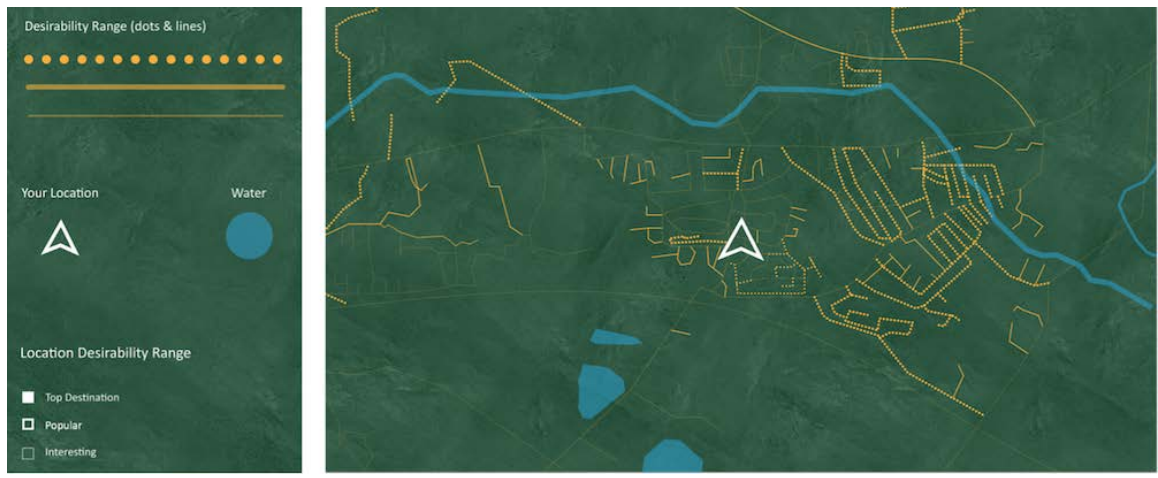

Figure 12. Paths of Desire Style sheet developed by Magnetic North

The paths and POI are both supported by a 3-level visual hierarchy based upon definitions under the control of the map designers. For example this could be based on predefined values to promote particular routes and POI, or calculated in real-time by monitoring the GPS-derived location of other app users in the city (i.e. with features classified as having the least visitors given hierarchical prominence). The screenshot on the left of Figure 13 is an early attempt to recreate the dots of style-sheet, which were not possible due to limitations in the vector-tile rendering software upon which the application is built. As a result of this, the current version of the application uses three different line weights for the visual hierarchy, as is illustrated in the screenshot to the right of Figure 13. Ultimately as an extension to this research we intend to create a new vector-tile map renderer that will allow us to fully realise the design, and have greater flexibility in the developments of future designs than is currently possible. 

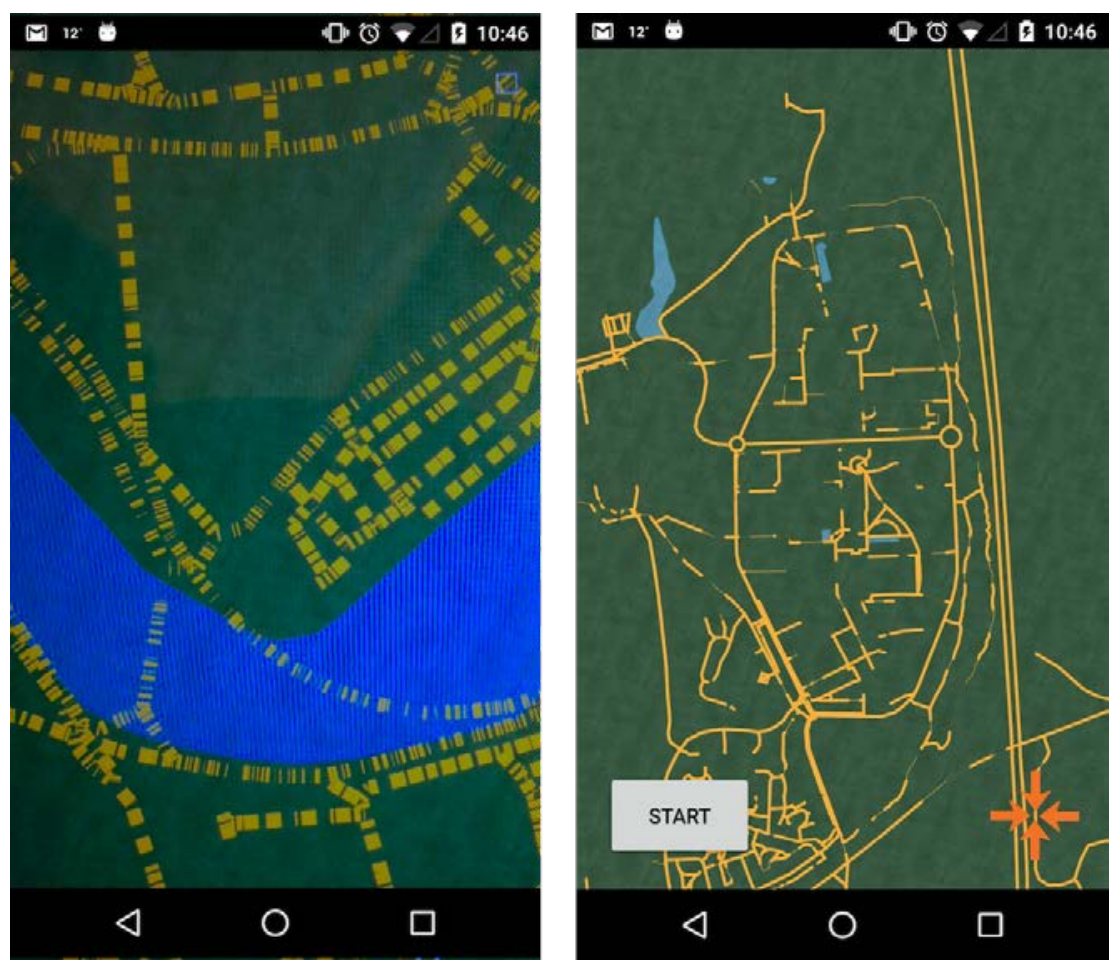

Figure 13. Paths of Desire applications screenshots

Once the basic principles of dynamic visual hierarchies had been demonstrated using the application and map described above, we were able to start developing new maps for use in the application. It is intended that such maps may be easily created for each city that the application is used in, in order that the map may reflect the 'feel' of the city itself, as well as providing a pleasing aesthetic that would encourage potential users to look at and interact with it, and promoting playful exploration. An example of such a map is the 'Lancaster Map' that is shown in Figure 14, which was developed in collaboration with the Lancaster-based artist Chas Jacobs ${ }^{13}$. Chas' art is well known around Lancaster, and often features the city itself, making him an ideal collaborator to achieve these design goals.

13 //www.chasjacobs.co.uk 


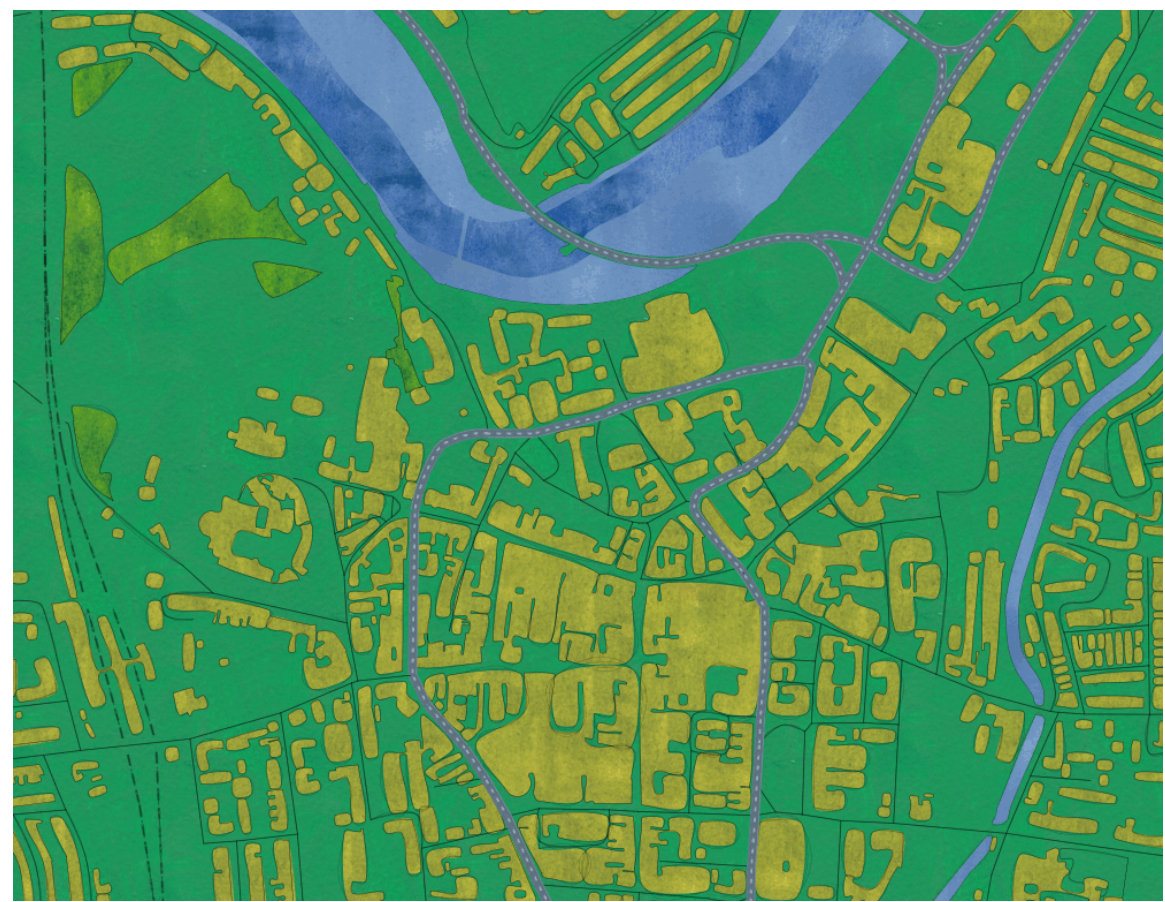

Figure 14. Screenshot of the 'Lancaster Map, developed in collaboration with Chas Jacobs.

Using approaches similar to those described the 'Sketchy Map' described above, the 'Lancaster Map' is designed to appear handdrawn, with polygon smoothing techniques used in order to achieve this, and give the appearance of pencil guide-lines around map features. The textures for the map were produced by Chas as squares of paint on plain paper, which were then scanned into digital imaging software and processed into seamless tiles that are then applied to the smoothed shapes as textures. An example of a painted square and the corresponding seamless tile that is used for the 'woodland' texture is given in Figure 15. 

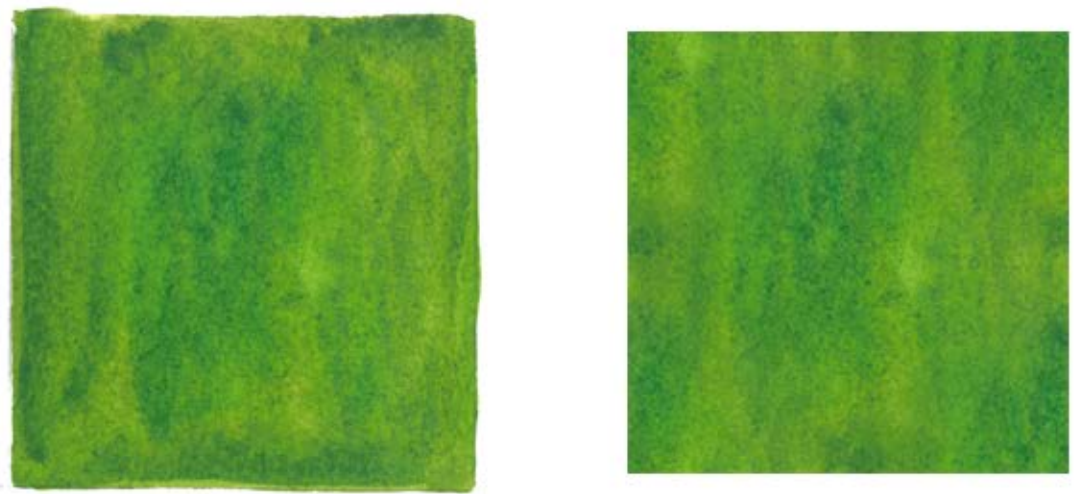

Figure 15. A square of paint produced by Chas Jacobs, and the corresponding seamless texture tile used in the map.

The maps themselves are implemented as a set of rule-based XML style-sheets that may either be stored locally on the mobile device or downloaded from the server as required. The style-sheets are then interpreted by the application renderer at run-time and styles are applied to features based upon the classification that is provided by the server. In this way, any number of optional maps may be implemented in the system for a variety of purposes, providing designs that suit the specific use-case of the application. For example, a map designed to guide tourists around historical attractions in a city might be antique in appearance, or one that it intended to facilitate playful exploration might look like a children's treasure map. In this way, the Paths of Desire application provides a novel approach to using cartography to affect behavior, as well as a mechanism by with new map designs can be deployed quickly and easily on mobile devices.

The ability to dynamically change the design of maps as a reaction to external factors has a number of implications for the future of the role of maps. Firstly, for the creation of the playable city in that dynamic in game events can be reflected directly upon the game map presented to the players, and secondly, it provides the ability to reconfigure the flow of people through a city in relation to particular requirements or events. 


\section{Discussion and Conclusions}

Taking inspiration from the Situationists, our consideration of the playable city draws upon their notions of detournement ('rerouting' or 'hijacking') and derive (an awareness of psychogeographical effects) in order to remap the city for play. Drawing upon the rich history of games and maps we have considered the playable city through the lens of neocartography. This approach recognises that whilst there can be little doubt that the widespread availability of digital and mobile maps has significantly impacted our interactions with and use of maps, these maps are being provided by a small number of commercial providers and exhibit limited opportunities for customization. This has resulted in the creation of a perceived 'blandscape', in which the traditional purpose-driven cartographic designs have taken a back seat to the application of recognizable commercial colour-schemes.

Whilst there have been calls for greater personalization of digital maps the resulting solutions have been directed at usability, efficiency, and clarity relative to the task being performed which has contributed to the homogenization of map aesthetics. It is unfortunate that this homogenization has also permeated through to the design of location based games which, in contrast with other types of computer game, have yet to realise the full potential of digital cartography.

In this research we have considered an alternative whereby the aesthetics of maps are used to affect the user experience in relation to exploration and serendipity within the playable city. Firstly, we have presented location based game that both adopts an aesthetic appropriate to the game but also uses abstraction of features on the digital map to encourage 'head-up' rather than 'head-down' navigation thus promoting users' engagement with their physical surroundings. Secondly, we have presented a system capable of producing digital maps whose design can be adapted dynamically and in particular in which the hierarchy of POI, roads and paths can be adjusted in real-time, with the aim of exploiting the known impact of map design upon behaviour in order to encourage users to explore their environment. 
Overall we believe that rich history of cartographic design has yet to be adequately addressed in relation to the aesthetics of digital maps, which we believe are an important component in realizing the playable city.

\section{Acknowledgments}

The research presented in this paper has been made possible through the Arts and Humanities Research Council (AHRC) project (AH/J005150/1) The Creative Exchange at Lancaster University in collaboration with Manchester design agency Magnetic North and the Lancaster based artist Chas Jacobs.

\section{References}

Ahlqvist, O. (2011). Converging themes in cartography and computer games. Cartography and Geographic Information Science, 38(3), pp 278-285.

Ashton, A. (2012) Sketchy Maps with Geometry Smoothing. https://www.mapbox.com/blog/sketchy-maps/. Last accessed 14/03/2016.

Ballatore, A and Bertolotto, M. (2015). Personalizing Maps. Communications of the ACM. Vol 58. No 12. pp 68-74.

BBC News (2008). Online maps 'wiping out history'. http://news.bbc.co.uk/1/hi/uk/7586789.stm, last accessed 14/03/2016.

Celino, I., Cerizza, D., Contessa, S., Corubolo, M., Dell'Aglio, D., Valle, E. D., and Fumeo, S. (2012). Urbanopoly--A Social and Location-Based Game with a Purpose to Crowdsource Your Urban Data. In Privacy, Security, Risk and Trust (PASSAT), 2012 International Conference on and 2012 International Confernece on Social Computing (SocialCom) (pp. 910913). IEEE.

Chehimi, F., Coulton, P., and Edwards, R. (2008). Using a camera phone as a mixed-reality laser cannon. International Journal of Computer Games Technology, 2008, 2.

Clark, J. (2008). The New Cartographers - What does it mean to map everything all the time?” In these times http://www.inthesetimes.com/article/3524/the new cartographers, last accessed $14 / 03 / 2016$. 
Coulton, P., Rashid, O., and Bamford, W. (2006). Experiencing 'touch' in mobile mixed reality games. In Proceedings of International Conference in Computer Game Design and Technology.

Coulton, P., Bamford, W., Cheverst, K., and Rashid, O. (2008). 3D Space-time visualization of player behaviour in pervasive location-based games. International Journal of Computer Games Technology, 2008, 2.

Culin, S. (1891). Street Games of Boys in Brooklyn, NY. The Journal of American Folklore, 4(14), pp 221-237.

Debord, G. (1955). Introduction to a critique of urban cartography. Situationist International Online, Translated by Ken Knabb, http://www.cddc.vt.edu/sionline/presitu/geography.html, last accessed 14/03/2016.

Demaj, D., and Field, K. (2012). Reasserting design relevance in cartography: some examples. The Cartographic Journal, 49(1), pp 77-93.

Deterding, S., Sicart, M., Nacke, L., O'Hara, K., and Dixon, D. (2011). Gamification. using game-design elements in non-gaming contexts. In CHI'11 Extended Abstracts on Human Factors in Computing Systems (pp. 2425-2428). ACM.

Field, K., and Demaj, D. (2012). Reasserting design relevance in cartography: some concepts. The Cartographic Journal, 49(1), pp 70-76.

Field, K. (2013). Editorial. Cartomyopic musings. The Cartographic Journal, 50(1), pp 1-7.

Garfield, S (2012). On the map: Why the world looks the way it does. London, Profile.

Gill, G. (1993). Road map design and route selection. The Cartographic Journal, 30(2), pp 163166.

Gradinar, A., Huck, J., Coulton, P., Lochrie, M., and Tsekleves, E. (2015). Designing for the dichotomy of immersion in location based games. In proceedings of Foundations of Digital Games 2015.

Gradinar, A., Huck, J., Coulton, P., \& Salinas, L. (2016). Beyond the blandscape: utilizing aesthetics in digital cartography. In Proceedings of the 2016 CHI Conference Extended Abstracts on Human Factors in Computing Systems, pp. 1383-1388).

Huck, J., Coulton, P., Gradinar, A., and Whyatt, D. (2015). Cartography, location-based gaming and the legibility of mixed reality spaces. In proceedings of $23^{\text {rd }}$ GIS Research UK Conference.

Huck, J., Gradinar, A., Coulton, P., and Salinas, L. (2016). Paths of Desire: Dynamic Visual Hierarchies to Intentionally Influence Route Decision. In proceedings of $24^{\text {th }}$ GIS Research UK Conference.

Jenks, G. F. (1963). Generalization in statistical mapping. Annals of the Association of American Geographers, 53(1), pp 15-26.

Kent, A. J. (2008). Cartographic blandscapes and the new noise: Finding the good view in a topographical mashup. The Bulletin of the Society of Cartographers, 42(1),pp 29-37. 
Kraak, M. J. (2011). Is there a need for neo-cartography?. Cartography and Geographic Information Science, 38(2), pp 73-78.

Lammes, S. (2011). The map as playground: location-based games as cartographical practices'. Think, Design, Play, pp 1-10.

Lefebvre, H. (1991). The production of space (Vol. 142). Blackwell: Oxford.

Lei, Z., and Coulton, P. (2011). Using deliberate ambiguity of the information economy in the design of a mobile location based games. In Proceedings of the 15th International Academic MindTrek Conference: Envisioning Future Media Environments (pp. 33-36). ACM.

Licoppe, C., and Inada, Y. (2008). Geolocalized technologies, location-aware communities, and personal territories: the Mogi case. Journal of Urban technology, 15(3), pp 5-24.

McMaster, R., and McMaster, S. (2002). A history of twentieth-century American academic cartography. Cartography and Geographic Information Science, 29(3), pp 305-321.

Muehlenhaus, I. (2013). Web cartography: map design for interactive and mobile devices. CRC Press.

Rashid, O., Coulton, P., and Edwards, R. (2005). Implementing location based information/advertising for existing mobile phone users in indoor/urban environments. In Mobile Business, 2005. ICMB 2005. International Conference on (pp. 377-383). IEEE.

Rashid, O., Mullins, I., Coulton, P., and Edwards, R. (2006). Extending cyberspace: location based games using cellular phones. Computers in Entertainment (CIE), 4(1), 4.

Rashid, O., Bamford, W., Coulton, P., Edwards, R., and Scheible, J. (2006a). PAC-LAN: mixedreality gaming with RFID-enabled mobile phones. Computers in Entertainment (CIE), 4(4), 4.

Shepard, B., and Smithsimon, G. (2011). Beach Beneath the Streets, The: Contesting New York City's Public Spaces. SUNY Press.

Sotamaa, O. (2010). When the game is not enough: Motivations and practices among computer game modding culture. Games and Culture.

Tuters, M. (2011). Forget Psychogeography: The Object-Turn in Locative Media. In Conference Media in Transition (Vol. 7).

Tuters, M., and Varnelis, K. (2006). Beyond locative media: Giving shape to the internet of things. Leonardo, 39(4), 357-363.

Wark, M. (2015). The Beach Beneath the Street: The Everyday Life and Glorious Times of the Situationist International. Verso Books.

Whitehill, B. (1999). American Games: a historical perspective. Board Game Studies, 2(1), 11614. 
Wilson, D., Bertolotto, M., and Weakliam, J. (2010). Personalizing map content to improve task completion efficiency. International Journal of Geographical Information Science, 24(5), pp 741-760.

Wood, D. (2003). Cartography is dead (thank God!). Cartographic Perspectives, (45), pp 4-7.

Wright, J. K. (1942). Map makers are human: Comments on the subjective in maps. Geographical review, 32(4), pp 527-544.

Visvalingam, M., \& Whyatt, J. D. (1993). Line generalisation by repeated elimination of points. The Cartographic Journal, 30(1), pp 46-51. 